\title{
PRF Domain Extension Using DAGs
}

\author{
Charanjit S. Jutla \\ IBM T. J. Watson Research Center, \\ Yorktown Heights, NY 10598
}

\begin{abstract}
We prove a general domain extension theorem for pseudorandom functions (PRFs). Given a PRF $F$ from $n$ bits to $n$ bits, it is well known that employing $F$ in a chaining mode (CBC-MAC) yields a $\mathrm{PRF}$ on a bigger domain of $m n$ bits. One can view each application of $F$ in this chaining mode to be a node in a graph, and the chaining as edges between the node. The resulting graph is just a line graph. In this paper, we show that the underlying graph can be an arbitrary directed acyclic graph (DAG), and the resulting function on the larger domain is still a PRF. The only requirement on the graph is that it have unique source and sink nodes, and no two nodes have the same set of incident nodes. A new highly parallelizable MAC construction follows which has a critical path of only $3+\log ^{*} m$ applications of $F$.

If we allow Galois field arithmetic, we can consider edge-colored DAGs, where the colors represent multiplication in the field by the color. We prove an even more general theorem, where the only restriction on the colored DAGs is that if two nodes ( $u$ and $v$ ) have the same set of incident nodes $W$, then at least one $w$ in $W$ is incident on $u$ and $v$ with a different colored edge. PMAC (Parallelizable Message Authentication [6]) is a simple example of such graphs. Finally, to handle variable length domain extension, we extend our theorem to a collection of DAGs. The general theorem allows one to have further optimizations over PMAC, and many modes which deal with variable lengths.
\end{abstract}

Keywords: PRF, MAC, DAG, Partial Order, Galois Field.

\section{Introduction}

There is often a need to extend the domain of a given pseudo-random function (PRF). One of the most popular and well-known such schemes is the CBCMAC [1]. In [3] it was shown that if $F$ is a pseudo-random function from $n$ bits to $n$ bits, then the CBC (cipher block chaining) construction yields a PRF from $m n$ bits to $n$ bits. Although the construction is called a MAC (message authentication code), which is a strictly weaker notion than PRF [9], the above shows that it is indeed a PRF domain extension method. Other domain extension schemes are known as well, for example, the cascade construction [2] and the protected counter sum construction [5]. Recently, a scheme PMAC (or Parallelizable Message Authentication) [6] (also see XECB [11]) was also shown to be a domain extension scheme. 
Despite all these results, there is no unifying theme in these results. In this paper, we attempt to remedy this situation, by proving a general theorem for domain extension. In essence, we show that arbitrary acyclic networks of the same pseudo-random function can be used to build a pseudo-random function on a larger domain. To illustrate this paradigm, consider the CBC-MAC scheme. Let $F$ be a PRF from $n$ bits to $n$ bits (and which takes $k$ bits of secret key). For example, DES [10] is usually assumed to be such a PRF on 64 bits, with 56 bits of secret key. A PRF $\tilde{F}$ from $m n$ bits to $n$ bits is defined as follows. The $m n$ bit input is divided into $m$ blocks $P_{1}, P_{2}, \ldots, P_{m}$. The function $F_{k}$ (i.e. $F$ with key $k$ ) is applied to the first block $P_{1}$ to yield an intermediate value $C_{1}$. The function $F_{k}$ is next invoked on the xor of the next block $P_{2}$ and previous intermediate value $C_{1}$, to yield $C_{2}$. This chaining process is continued, and the output of $\tilde{F}_{k}$ is just $C_{m}$. The chaining process defines an underlying directed graph of $m$ nodes $V_{1}, V_{2}, \ldots, V_{m}$, with an edge from $V_{i}$ to $V_{i+1}$.

Now, consider an arbitrary directed acyclic graph (DAG) $G=(V, E)$, with $m$ nodes $V$, and edges $E$. Assume that $G$ has only one source node $V_{1}$, and only one sink node $V_{m}$. Given a PRF $F$ from $n$ bits to $n$ bits, a composite PRF $\tilde{F}$ from $m n$ bits to $n$ bits is defined as follows. As before, assume that the input is a sequence $P_{1}, \ldots, P_{m}$. The first intermediate value is just $C_{1}=F_{k}\left(P_{1}\right)$. Inductively assume that we have computed the intermediate values of all predecessors of a node $V_{i}$. Then, the intermediate value $C_{i}$ for the node $V_{i}$ is

$$
C_{i}=F_{k}\left(P_{i} \oplus \bigoplus_{(i, j) \in E} C_{j}\right)
$$

The output of the composite function $\tilde{F}_{k}$ is just $C_{m}$. See Figure 1 for an example.

Of course, not all DAGs are expected to yield a PRF. However, consider DAGs with the restriction that no two nodes have the same set of incident nodes

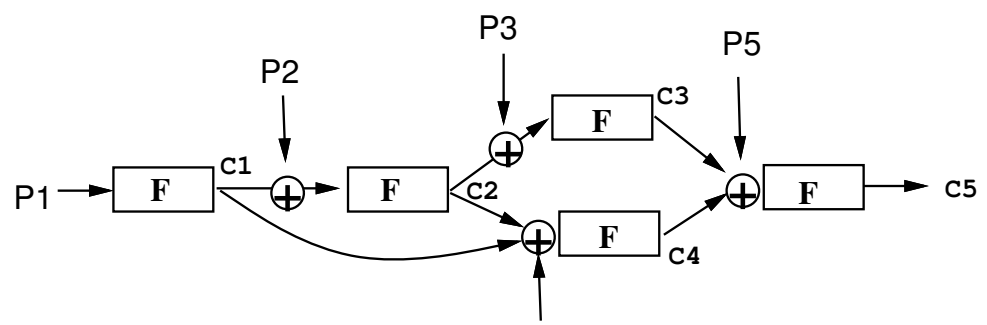

P4

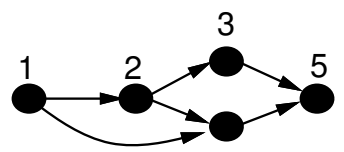

4

Fig. 1. A PRF Domain Extension Mode and its DAG 
( $u$ is said to be incident on $v$ if there is an edge from $u$ to $v$ ), and that they have unique source and sink nodes. In this paper we show that given a PRF $F$ from $n$ bits to $n$ bits, the composite $\tilde{F}$ defined as above on such DAGs, is a PRF from $m n$ bits to $n$ bits.

An immediate application is that if a party has access to parallel hardware, then instead of simple chaining as in CBC-MAC, it can compute the PRF in parallel. For instance, if it has four processors, then it can employ the method given by the graph in Figure 2. A parallel mode with critical path of length only $3+\log ^{*} m$ also follows. Unlike PMAC [6], this mode does not use any Galois arithmetic.

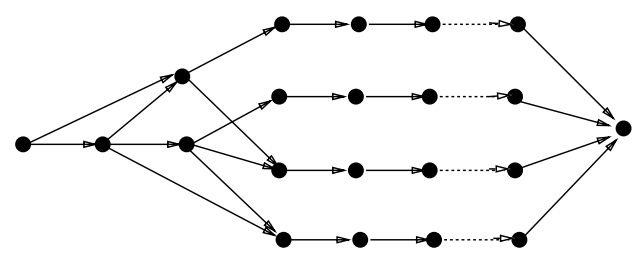

Fig. 2. A Parallel Mode for four processors

If we allow Galois Field arithmetic (in particular, fields $\mathrm{GF}\left(2^{n}\right)$ ), we can consider edge-colored DAGs. The colors on the edges represent multiplication in the field by the color (assume that each color is mapped to a unique element in the field). For example, going back to figure 1, suppose we employ three colors, col1, col2 , and col3. Let $w$ be a primitive element in the field. We map col1 to unity in the field, col2 to $w$, and col3 to $w^{2}$. Then, if we color the edge $(1,4)$ by col2, then in the definition of the composite function, we multiply the intermediate result $C_{1}$ with $w$ in the field, before xoring it with the plaintext $P_{4}$ and $C_{2}$, and applying $F_{k}$.

The main result of the paper can be stated as follows. Consider an edge colored DAG $G$ with unique source and sink nodes and $m$ total nodes, and with the condition that if two nodes (say $u$ and $v$ ) have the same set of incident nodes (say $W$ ), then for at least one node $w$ in $W$, the color on the edge $(w, u)$ is different from the color on the edge $(w, v)$. Given a PRF $F$ from $n$ bits to $n$ bits, the composite $\tilde{F}$ built using the graph $G$ as above, is a PRF from $m n$ bits to $n$ bits. The result is proven under the adaptive adversary model, which is of course the difficult case. Our proof technique is novel, and even when considered as just a proof for CBC-MAC it offers a simpler and novel proof in the adaptive adversary model. It is well known that the difficulty in analyzing the security of such schemes stems from the fact that we need to model the underlying oracle as a function, i.e. an oracle replying consistently with earlier queries. The key advance is an identity (lemma 1) which reduces the analysis to a scheme where the oracle replies randomly. The adversary remains adaptive, but the analysis in this "random game" becomes much easier.

Using the new theorem, the mode in fig 2 can now be parallelized further as in fig 3(a). The additional cost is a few $\operatorname{GF}\left(2^{n}\right)$ operations. Security of PMAC [6] 


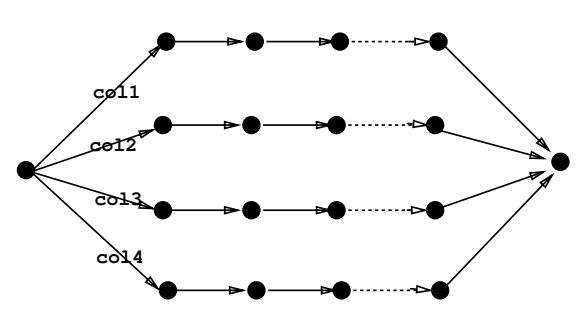

(a)

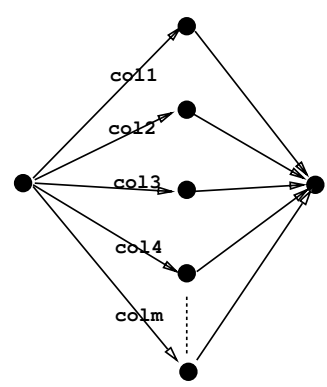

(b)

Fig. 3. Modes using $\mathrm{GF}\left(2^{n}\right)$

follows (see fig 3(b)), as it is a simple example of such a colored DAG. Further, we obtain an additional optimization over PMAC, because unlike PMAC, we do not even need to compute $F_{k}$ on the all zero word (i.e. $F_{k}\left(0^{n}\right)$ ).

In Section 5 we extend our results to variable length domain extension.

\section{Definitions}

Definition 1. For positive integers $n$, $l$, let $\mathcal{F}(n \rightarrow l)$ be the set of all functions from $n$ bits to $l$ bits.

Definition 2. (PRF) A pseudo-random function has signature

$$
F:\{0,1\}^{k} \times\{0,1\}^{n} \rightarrow\{0,1\}^{l} .
$$

Define $\operatorname{Sec}_{F}(q, T)$ to be the maximum advantage an adaptive adversary can obtain when trying to distinguish between $F_{K}(\cdot)$ (with $K$ chosen uniformly at random) and a function chosen uniformly at random from $\mathcal{F}(n \rightarrow l)$, when given $q$ queries and time $T$.

\section{Domain Extension Using Arbitrary Acyclic Graphs}

Definition 3. Let $G=(V, E)$, be a directed acyclic graph (DAG) [13] with a finite vertex set $V$ and edges $E$. A node $u$ is said to be incident on a node $v$, if there is an edge from $u$ to $v$, i.e $E(u, v)$. Such an edge will sometimes be denoted $\langle u, v\rangle$. Define a DAG to be non-redundant if for every pair of nodes, the set of their incident nodes is different. For two vertices $u$ and $v$, we say that $u \prec v$ if there is a directed path from $u$ to $v$. Since $G$ is a finite DAG, the relation $\prec$ is a finite partial order.

Definition 4. Given a function $f$ from $n$ bits to $n$ bits, and a non-redundant DAG $G=(V, E)$ with only one source node and only one sink node, and a total of $m$ nodes, define $f^{G}:\{0,1\}^{n m} \rightarrow\{0,1\}^{n}$ as follows: 
- Let the input to $f^{G}$ be an $m n$ bit string $P$, which is divided into $m n$-bit strings $P_{1}, P_{2}, \ldots, P_{m}$.

- Since $|V|=m$, let $V_{1}, \ldots ., V_{m}$ be an enumeration of the nodes. When it is clear from context, we will identify the index of a vertex with the vertex itself. Let the unique source node be $V_{1}$, and the unique sink node be $V_{m}$.

- For the unique source node, define $M_{1}=P_{1}$.

- For every non-source node $V_{j}, j>1$, inductively (over $\prec$ ) define $M_{j}=$ $P_{j} \oplus_{u: E(u, j)} f\left(M_{u}\right)$

- For notational convenience, for every node $V_{j}$, let $C_{j}$ denote $f\left(M_{j}\right)$.

- The output of the function $f^{G}$ is just $C_{m}$.

It is clear that the restriction of one sink node is crucial, for if there was another sink node other than $V_{m}$, then the plaintext fed into this other sink node has no influence on $C_{m}$. It is possible that there are instances of DAGs $G$ with two source nodes such that $F^{G}$ is a PRF; however, a more stringent requirement than non-redundancy will definitely be required. Consider a DAG $G$, with two source nodes $V_{1}$ and $V_{2}$, both with only one outgoing edge and that too to the same vertex. Then, the resulting function is clearly not a PRF. A similar situation motivates the requirement of non-redundancy.

One may be tempted to weaken the non-redundancy requirement. For instance, one idea is to have a condition on the DAG that it have no non-trivial automorphism. However, such a DAG may not yield a secure PRF, as illustrated in Figure 4. The two queries $\langle p 1, p 2, p 2, p 4, p 5, p 6\rangle$ and $\langle p 1, p 2, p 2, p 5, p 4, p 6\rangle$ yield the same result.

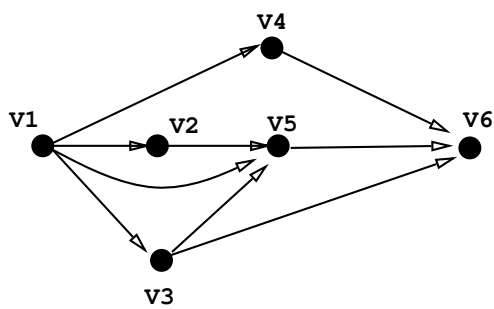

Fig. 4. A non-automorphic DAG

Theorem 1. For a non-redundant $D A G G=(V, E)$ with unique source and sink nodes, and $m$ total nodes, let $f^{G}$ be as above. Then, no adaptive adversary, with $q$ queries, can distinguish between (a) $f^{G}$ where $f$ is chosen uniformly at random from $\mathcal{F}(n \rightarrow n)$, (b) and a function chosen uniformly at random from $\mathcal{F}(n m \rightarrow n)$, with probability more than $(m q)^{2} 2^{-(n+1)}$.

In the next section, we state and prove a more general theorem.

\section{Domain Extension Using Colored DAGs and GF $\left(2^{n}\right)$}

If we allow Galois field arithmetic, we get an even more general construction, and a corresponding PRF domain extension theorem. Assuming that the 
underlying function $F$ has an $n$-bit output, we will use the Galois field $\operatorname{GF}\left(2^{n}\right)$. Such fields have the property that they have exactly $2^{n}$ elements. Moreover, each element can be represented as a $n$ bit vector, with addition in the field being just the bitwise exclusive-or $(\oplus)$. Since multiplication distributes over addition in a field, it follows that if $a, b$ and $c$ are three elements in the field then $a *(b \oplus c)=a *(b+c)=(a * b)+(a * c)=(a * b) \oplus(a * c)$. A further useful property of finite fields is that for a fixed non-zero $a$ in the field, if $b$ is picked uniformly at random from the field, then $a * b$ is also uniformly distributed in the field.

Definition 5. Let $G=(V, E)$, be a directed acyclic graph (DAG). Let $|V|=m$. A coloring $\chi$ of the edges of the graph is a map $\chi: E \rightarrow[1 . . m]$. The triple $(V, E, \chi)$ will be called an edge-colored DAG. Define an edge-colored DAG to be nonsingular if for every pair of nodes $u, v$, if the set of their incident nodes is same (say $W)$, then at least for one $w \in W, \chi(\langle w, u\rangle) \neq \chi(\langle w, v\rangle)$. For two vertices $u$ and $v$, we say that $u \prec v$ if there is a directed path from $u$ to $v$. Since $G$ is a finite $\mathrm{DAG}$, the relation $\prec$ is a finite partial order.

Definition 6. Given a function $f$ from $n$ bits to $n$ bits, and a non-singular edge-colored DAG $G=(V, E, \chi)$ with only one source node and only one sink node and a total of $m<2^{n}$ nodes, define $f^{G}:\{0,1\}^{n m} \rightarrow\{0,1\}^{n}$ as follows:

- Since $m<2^{n}$, we can view $\chi$ as a map from $E$ to $\operatorname{GF}\left(2^{n}\right)^{*}$, i.e. the non- zero elements of the field.

- Let the input to $f^{G}$ be $m n$ bit string $P$, which is divided into $m n$-bit strings $P_{1}, P_{2}, \ldots, P_{m}$.

- Since $|V|=m$, let $V_{1}, \ldots ., V_{m}$ be an enumeration of the nodes. When it is clear from context, we will identify the index of a vertex with the vertex itself. Let the unique source node be $V_{1}$, and the unique sink node be $V_{m}$.

- For the unique source node, define $M_{1}=P_{1}$.

- For every non-source node $V_{j}, j>1$, inductively (over $\prec$ ) define $M_{j}=$ $P_{j}+\sum_{u: E(u, j)} \chi(\langle u, j\rangle) * f\left(M_{u}\right)$, where $f\left(M_{u}\right)$, which is an $n$-bit quantity, is viewed as an element of $\operatorname{GF}\left(2^{n}\right)$. The summation is addition in the field, which is the same as $n$-bit exclusive-or.

- For notational convenience, for every $j$, we denote $f\left(M_{j}\right)$ by $C_{j}$.

- The output of the function $f^{G}$ is just $C_{m}$.

Theorem 2. : (Main Theorem) For a non-singular edge-colored DAG $G=$ $(V, E, \chi)$ with unique source and sink nodes, and $m<2^{n}$ total nodes, let $f^{G}$ be as above. Then, no adaptive adversary, with $q$ queries, can distinguish between (a) $f^{G}$ where $f$ is chosen uniformly at random from $\mathcal{F}(n \rightarrow n)$, (b) and a function chosen uniformly at random from $\mathcal{F}(n m \rightarrow n)$, with probability more than $(m q)^{2} 2^{-(n+1)}$.

Theorem 3. Given a PRF F: $\{0,1\}^{k} \times\{0,1\}^{n} \rightarrow\{0,1\}^{n}$, and a non-singular edge-colored $D A G G=(V, E, \chi)$ with unique source and sink nodes, and $m<2^{n}$ total nodes, a function $F^{G}:\{0,1\}^{k} \times\{0,1\}^{m n} \rightarrow\{0,1\}^{n}$ can be defined be letting for each $K,\left(F^{G}\right)_{K}$ to be $\left(F_{K}\right)^{G}$ (as in definition 6$)$. Then, 


$$
\operatorname{Sec}_{F^{G}}(q, T) \leq \operatorname{Sec}_{F}(q, T)+(m q)^{2} 2^{-(n+1)}
$$

The proof follows from Theorem 2 by standard techniques.

\subsection{Background}

Most theorems in cryptography involving PRF [3,2,5,6,11] and PRP $[21,22,15,12]$ constructions, as well as modes of operations of block ciphers $[4,16,11,24,19]^{1}$, from other primitives must tackle the issue of collisions in the oracle calls to the smaller primitive. Fortunately, these collision probabilities are usually low, and conditioning on distinctness of oracle calls, the target construct can be shown to behave like a random function or permutation.

Upper bounding the collision probability requires different techniques in many of these theorems, and the difficulty in the proof can depend on issues like whether there are two independent oracles (as in $[22,16]$ ) or if a fresh initial vector is used in each invocation (as in $[4,16,24,19])$. In particular, the proof of security of CBC-MAC [3] is more involved than that of CBC [4] for precisely this reason, i.e. in the former there is no fresh initial vector in each invocation. As our theorem generalizes CBC-MAC, we expect similar intricacies in our proof. However, as mentioned in the introduction, we prove a novel technical lemma which precisely captures this nuance of CBC-MAC.

\subsection{Notation}

Before we prove theorem 2, we need to fix more notation and give a general idea of the proof. We first note that we allow arbitrary functions as adversaries and not just computable functions. Then without loss of generality, we can assume that the adversary is deterministic, as every probabilistic adversary is just a probability distribution over all deterministic adversaries [18].

Fix an adaptive adversary. Since the adversary is deterministic, the first query's plaintext (say $P^{1}=\left\langle P_{1}^{1}, \ldots, P_{m}^{1}\right\rangle$ ) is fixed for that adversary. Thus, the first query's output, say $C_{m}^{1}$ is only a function of $f$. The adversary being adaptive, its second query is a function of $C_{m}^{1}$. But, since $C_{m}^{1}$ is only a function of $f$, the second query's plaintext can also be written just as a function of $f$. Thus, $C_{m}^{2}$ is only a function of $f$, and so forth.

We will denote probabilities under the first scenario, i.e. (a) in the theorem 2 statement, as $\operatorname{Pr}$, and the probabilities in the second scenario, i.e. (b) in the theorem 2 statement, as $\operatorname{Pr}_{(b)}$. Most of the analysis will be devoted to the first scenario. So, unless otherwise mentioned, all random variables from now on are in the first scenario.

For all variables corresponding to a query, we will use superscripts to denote the query number. Subscripts will be used to denote blocks within a query. The variables will be as in Definition 6, i.e. $P$ standing for plaintext input, $M$ standing for the variable on which the $f$ function is applied, and $C$ standing for the output of $f$.

\footnotetext{
${ }^{1}$ These references are not meant to be exhaustive.
} 
Thus, by the convention above $C_{j}^{i}$ is the output of $f$ in the $i^{\text {th }}$ query's $j^{\text {th }}$ block. We will use $C$ to denote the whole transcript $\left\{C_{j}^{i}\right\}_{i \in[q], j \in[m]}$ of $f$ outputs. There will often be a need to just refer to the sequence of last blocks of each query; we will use $C_{m}^{*}$ to denote the sequence $C_{m}^{1}, \ldots, C_{m}^{q}$, i.e. the $m$ th block from all the queries. More precisely, as argued earlier, these variables should be written as a function of $f$, e.g. $C(f)$, but we will drop the argument when it is clear from context.

Let $c$ denote a constant $m q n$-bit transcript, i.e. a prospective value for $C(f)$. For a fixed $c, P_{j}^{i}$ and $M_{j}^{i}$ can be viewed as functions of only $c$ (see definition 6 ), and we will write them as $P_{j}^{i}(c)$ and $M_{j}^{i}(c)$. Just as for $C$, we will use $P(c)$ to denote the whole sequence.

Definition 7. Given a constant $m q n$-bit transcript $c$, let the plaintext chosen by the adversary be $p=P(c)$. For any vertices $j, j^{\prime}$, and query indices $i, i^{\prime}$ we say that $(i, j) \equiv_{c}\left(i^{\prime}, j^{\prime}\right)$ if

$$
\left(j=j^{\prime}\right) \text { and } \forall k \preceq j: p_{k}^{i}=p_{k}^{i^{\prime}}
$$

Define

$$
\mu_{c}(i, j)=\min \left\{i^{\prime} \mid\left(i^{\prime}, j\right) \equiv_{c}(i, j)\right\}
$$

Not every $m q n$-bit constant $c$ can be a real transcript $C(f)$ for some $f$. So, we define a notion of consistent $c$. We call $c$ consistent $(\operatorname{con}(c))$ if

$$
\forall j \in[1 . . m], \forall i: c_{j}^{i}=c_{j}^{\mu_{c}(i, j)}
$$

Define the following "correcting" function $\rho$ from $m q n$-bit blocks to $m q n$-bit blocks:

$$
\rho(c)=\bar{c}, \text { where } \bar{c}_{j}^{i}=c_{j}^{\mu_{c}(i, j)} .
$$

The above definition of a correcting function is similar to the one used in the proof of the Luby-Rackoff theorem (see [20]).

Define the core index set of $c$ to be $I=\left\{(i, j) \mid \mu_{c}(i, j)=i\right\}$. Informally, $I$ is the set of indices which are not required to be "consistent" with smaller indices. Thus, $\rho(c)$ retains the values at core indices, and corrects them otherwise.

Consider the following condition PD (pairwise different).

Definition 8. For any constant $c$, define $\mathrm{PD}(c)$ to be

$$
\begin{gathered}
\forall i, i^{\prime} \in[1 . . q], \forall j, j^{\prime} \in[1 . . m], j \neq j^{\prime}: M_{j}^{i}(c) \neq M_{j^{\prime}}^{i^{\prime}}(c), \\
\text { and } \forall i, i^{\prime} \in[1 . . q], \forall j \in[1 . . m]:(i, j) \not \equiv_{c}\left(i^{\prime}, j\right) \Rightarrow M_{j}^{i}(c) \neq M_{j}^{i^{\prime}}(c) \text {. }
\end{gathered}
$$

\subsection{Proof of Main Theorem}

Lemma 1 (PRF Technical Lemma). For every $q n$-bit constant $r=\left\langle r^{i}\right\rangle_{i \in[1 . . q]}$

$$
\begin{aligned}
& \operatorname{Pr}_{c \in_{U}\{0,1\}^{m q n}, f \in_{U}} \mathcal{F}_{(n \rightarrow n)}\left[C(f)=c \wedge P D(c) \mid c_{m}^{*}=r\right] \\
& =2^{-m q n} * P r_{c \in U}\{0,1\}^{m q n}\left[P D(\rho(c)) \mid c_{m}^{*}=r\right]
\end{aligned}
$$


In the left hand side of the lemma, we have that $c$ is consistent, as it is not difficult to see that $C(f)$ is consistent (as proven below in lemma 2(i)). Now for consistent $c$, it is also easy to see that "correcting" it leaves it unchanged, i.e. $\rho(c)=c$ (see lemma $2(\mathrm{f}))$. Hence, we can replace $\operatorname{PD}(c)$ by $\operatorname{PD}(\rho(c))$ in the left hand side above. Thus, the lemma can be restated as

$$
\operatorname{Pr}_{c, f}\left[C(f)=c \mid \operatorname{PD}(\rho(c)) \wedge c_{m}^{*}=r\right]=2^{-m q n}
$$

To prove this, we can try to see what constraints are imposed on $f$ and $c$ by $C(f)=c$. For $C(f)$ to be same as $c$, the transcript $c$ must be consistent, as $C(f)$ is consistent. Let $I$ be the core index set of $c$. Let $l=|I|$. Then for $c$ to be consistent, there are exactly $(m q-l) n$-bit linear constraints on $c$. We will also see that for every consistent $c$ (in which case $c=\rho(c)$ ), such that $P D(c)$ holds, a function $f_{c}$ can be defined using the $M$ and the $c$ values at core indices $I$ such that $C\left(f_{c}\right)=c$ (see lemma 3 ). Moreover, such an $f_{c}$ is unique on these $l$ input values $M$ (lemma $2(\mathrm{f}))$. Thus, there are exactly $l$ n-bit constraints on $f$ such that $C(f)=c$. Thus, there are a total of $m q$ n-bit constraints on $f$ and $c$. However, we have not addressed the issue of whether the condition $\operatorname{PD}(\rho(c))$ perhaps influenced this count of $m q$ total constraints. We show below rigorously that even under the condition $\operatorname{PD}(\rho(c))$ the number of $n$-bit constraints on $f$ and $c$ is exactly $m q$.

So to start with, for each consistent $c$, we would like to define a function $f_{c}$ such that $C\left(f_{c}\right)=c$. We also show below (lemma $2(\mathrm{~h})$ ) that for consistent $c$, $M_{j}^{i}(c)=M_{j}^{\mu_{c}(i, j)}(c)$. Thus, if we define $f_{c}$ at core indices, i.e. define $f_{c}\left(M_{j}^{i}(c)\right)=$ $c_{j}^{i}$ for all $i$ in $I$, we might have $C\left(f_{c}\right)=c$. There is a slight problem however, i.e. $f_{c}$ may not be well-defined, as the $M(c)$ values at core indices may not be distinct. In fact, we will need an even stronger distinctness condition, i.e. PD defined above, than just being distinct at core indices.

Definition 9. For each $c$, such that $\mathrm{PD}(c)$ holds, define $f_{c}$ as follows. Let $I=$ $\left\{(i, j) \mid \mu_{c}(i, j)=i\right\}$ be the core index set. For $(i, j) \in I$, define $f_{c}\left(M_{j}^{i}(c)\right)=c_{j}^{i}$. This is well defined as $\mathrm{PD}(c)$ holds. We will not need to define $f_{c}$ on other values.

In Lemma 3 below we show that for every consistent $c$ such that $\mathrm{PD}(c)$ holds it is indeed the case that $C\left(f_{c}\right)=c$.

We collect all simple statements about $\mu, \rho$ and consistency and their relationships to each other in the following lemma.

Lemma 2. For all $i, i^{\prime} \in[1 . . q], i \neq i^{\prime}$, for all $j \in[1 . . m]$ and mqn bit constant transcript $c$ :

(a) $(i, m) \not \equiv_{c}\left(i^{\prime}, m\right)$, i.e. $\mu_{c}(i, m)=i$,

(b) $\equiv_{c}$ is an equivalence relation,

(c) $\mu_{c}\left(\mu_{c}(i, j), j\right)=\mu_{c}(i, j)$,

(d) $\mu_{c}=\mu_{\rho(c)}$,

(e) $\rho(c)$ is consistent,

(f) Let $c$ be consistent, and let $b$ be such that for all $i$ s.t. $\mu_{c}(i, j)=i, b_{j}^{i}=c_{j}^{i}$.

Then $\rho(b)=c$. Also, for consistent $c, \rho(c)=c$ 
(g) For $u \preceq j, \mu_{c}(i, u)=\mu_{c}\left(\mu_{c}(i, j), u\right)$.

(h) For consistent $c, M_{j}^{i}(c)=M_{j}^{\mu_{c}(i, j)}(c)$

(i) $C(f)$ is consistent,

(j) For the transcript $c$ let $p=P(c)$ be its corresponding plaintext. If for all $u$

s.t. $E(u, j), \mu_{c}(i, u)=\mu_{c}\left(i^{\prime}, u\right)$, and $p_{j}^{i}=p_{j}^{i^{\prime}}$, then $\mu_{c}(i, j)=\mu_{c}\left(i^{\prime}, j\right)$.

Proof: (a) As we have assumed, wlog, that the adversary does not repeat queries, it follows that $i$ and $i^{\prime}\left(i \neq i^{\prime}\right)$ can never be equivalent over all vertices $V$. In particular, it is not the case that $(i, m) \equiv{ }_{c}\left(i^{\prime}, m\right)$. To see this, note that we have assumed that the graph has only one sink node, i.e. $V_{m}$. It follows that for every node $j, j \preceq m$, hence the claim.

(b) \& (c) straightforward.

(d) Note that the adversary's choice of $p=P(c)$ depends only on $c_{m}^{*}$. So we first show that for all $i, \rho(c)_{m}^{i}=c_{m}^{i}$. This follows as $\mu_{c}(i, m)=i$ by (a). Thus $p$ remains same for $\rho(c)$.

(e) We just note that for all $i, i^{\prime},(i, j) \equiv_{c}\left(i^{\prime}, j\right)$ implies $\mu_{c}(i, j)=\mu_{c}\left(i^{\prime}, j\right)$. Thus, by definition of $\rho$, we have $\rho(c)_{j}^{i}=\rho(c)_{j}^{i^{\prime}}$.

(f)We first note that, since by $(\mathrm{a}), \mu_{c}(i, m)=i$, we have $b_{m}^{i}=c_{m}^{i}$. Thus, as in proof of $(\mathrm{d})$ above, $\mu_{b}=\mu_{c}$. Now, $\rho(b)_{j}^{i}=b_{j}^{\mu_{b}(i, j)}=b_{j}^{\mu_{c, j}(i)}=c_{j}^{\mu_{c, j}(i)}$, the last equality following from (c) and condition on $b$. For consistent $c$, this is same as $c_{j}^{i}$. (g) For $u \preceq j,(i, j) \equiv_{c}\left(i^{\prime}, j\right)$ implies $(i, u) \equiv_{c}\left(i^{\prime}, u\right)$. Now, $(i, j) \equiv_{c}\left(\mu_{c}(i, j), j\right)$. Thus, $(i, u) \equiv_{c}\left(\mu_{c}(i, j), u\right)$.

(h) $M_{j}^{i}(c)=P_{j}^{i}(c)+\sum_{u: E(u, j)} \chi(\langle u, j\rangle) * c_{u}^{i}$. First note that $P_{j}^{i}(c)=P_{j}^{\mu_{c}(i, j)}(c)$. Also, for consistent $c$ and $u \preceq j, c_{u}^{i}=c_{u}^{\mu_{c}(i, u)}=c_{u}^{\mu_{c}\left(\mu_{c}(i, j), u\right)}$ by (g). Again by consistency of $c$, the latter is same as $c_{u}^{\mu_{c}(i, j)}$. This shows that $M_{j}^{i}(c)=$ $M_{j}^{\mu_{c}(i, j)}(c)$.

(i) by induction on the finite partial order $\prec$.

(j) We just need to show that $(i, j) \equiv_{c}\left(i^{\prime}, j\right)$. But $\mu_{c}(i, u)=\mu_{c}\left(i^{\prime}, u\right)$ implies $(i, u) \equiv_{c}\left(i^{\prime}, u\right)$. This along with $p_{j}^{i}=p_{j}^{i^{\prime}}$ shows that $p$ agrees in queries $i$ and $i^{\prime}$ over all blocks $j^{\prime} \preceq j$.

Lemma 3. For any consistent $c$ such that $P D(c)$ holds:

$$
C\left(f_{c}\right)=c
$$

Proof: Follows by induction. See the appendix for a full prove.

Lemma 1 (PRF Technical lemma restated). For every $q n$-bit constant $r=$ $\left\langle r^{i}\right\rangle_{i \in[1 . . q]}$

$$
\begin{aligned}
& \operatorname{Pr}_{c \in_{U}\{0,1\}^{m q n}, f \in_{U}} \mathcal{F}_{(n \rightarrow n)}\left[C(f)=c \wedge \operatorname{PD}(c) \mid c_{m}^{*}=r\right] \\
& =2^{-m q n} * \operatorname{Pr}_{c \in U}\{0,1\} m q n\left[\operatorname{PD}(\rho(c)) \mid c_{m}^{*}=r\right]
\end{aligned}
$$

Proof: We first show that the LHS above is same as

$$
\Gamma=\operatorname{Pr}_{c, b \in U}\{0,1\}^{m q n}\left[b_{j}^{i}=\left.c_{j}^{i}\right|_{(i, j): \mu_{c}(i, j)=i} \wedge \operatorname{con}(c) \wedge \operatorname{PD}(c) \mid c_{m}^{*}=r\right]
$$


By lemma 2(i), the conjunct $\operatorname{con}(c)$ can be added to the LHS of the lemma. We show that the two probabilities are same for every constant $c$. So, fix a $c$. As before, let $I=\left\{(i, j) \mid \mu_{c}(i, j)=i\right\}$ be the core index set of $c$. Let $S=$ $\left\{M_{j}^{i}(c) \mid(i, j) \in I\right\}$. Since $\mathrm{PD}(\mathrm{c})$ holds, $|S|=|I|$. Let $S^{\prime}$ be an arbitrary set of $n$ bit strings, disjoint from $S$, and $\left|S^{\prime}\right|=m q-|I|$. Thus, $\left|S \cup S^{\prime}\right|=m q$.

By lemma $3, C\left(f_{c}\right)=c$. Thus, for each $b$ agreeing with $c$ on $I$, we have a function $f_{c}$ defined on $|I|$ inputs $S$, such that $C\left(f_{c}\right)=c$. We can use the remaining $m q-|I|$ values of $b$ (i.e. from indices which are not in $I$ ) to extend $f_{c}$ to be defined on $S \cup S^{\prime}$. This map from $b$ to the extended $f_{c}$ is 1-1.

Similarly, for any function $f$ defined on $S \cup S^{\prime}$, such that $C(f)=c$ (note that $f$ need only be defined on $S$ for $C(f)$ to be well defined), we can define an $m q n$-bit long $b$ which agrees with $c$ on $I$. For indices in $(i, j) \in I$, use $f\left(M_{j}^{i}(c)\right)$ to define $b_{j}^{i}$, and use $f(s), s \in S^{\prime}$, to define the remaining part of $b$. This map from $f$ to $b$ is also $1-1$. This shows that the LHS of the statement of the lemma is same as $\Gamma$.

We next show that, the RHS of the statement of the lemma is same as $\Gamma$. To this end, we show that the following two sets are equinumerous, i.e. we show a bijection between the two sets. The two sets are

$$
\begin{gathered}
\mathcal{C}=\left\{c \mid c \in\{0,1\}^{m q n}, \operatorname{PD}(\rho(c)), \text { and } c_{m}^{*}=r\right\} \\
\mathcal{D}=\left\{(c, b)\left|c, b \in\{0,1\}^{m q n}, b_{j}^{i}=c_{j}^{i}\right|_{(i, j) \in I}, \operatorname{con}(c), \quad P D(c), \text { and } c_{m}^{*}=r\right\}
\end{gathered}
$$

That they are equinumerous follows easily from lemma $2(\mathrm{e}, \mathrm{f}, \mathrm{a}, \mathrm{d})$, but to be rigorous consider the following extension of $\rho$ to a function $\hat{\rho}$ from $\mathcal{C}$ to $\mathcal{D}$.

$$
\hat{\rho}(c)=(\rho(c), c)
$$

It needs to be shown that the function has $\mathcal{D}$ as its range, is 1-1 and onto. The function is obviously $1-1$. To prove that its range is $\mathcal{D}$, we need to prove three things:

1. $\rho(c)$ is consistent: follows by lemma $2(\mathrm{e})$.

2. $c_{j}^{i}=\left.\rho(c)_{j}^{i}\right|_{\mu_{\rho(c)}(i, j)=i}$ : follows directly from definition of $\rho$ and lemma $2(\mathrm{~d})$.

3. $\forall i, \rho(c)_{m}^{i}=r^{i}$ : by lemma $2\left(\right.$ a) and definition of $\rho$ we have $\rho(c)_{m}^{i}=c_{m}^{i}$; and hence $c_{m}^{i}=r^{i}$ implies $\rho(c)_{m}^{i}=r^{i}$.

To prove that it is onto, for any $(c, b)$ in $\mathcal{D}$, we show that $b$ is in $\mathcal{C}$ and $\hat{\rho}(b)=(c, b)$. But for any $(c, b)$ in $\mathcal{D}$, by lemma $2(\mathrm{f}), \rho(b)=c$. Thus, $\hat{\rho}(b)=(c, b)$. It also follows that $\mathrm{PD}(\rho(b))$ holds. Moreover, by lemma $2(\mathrm{a}), b_{m}^{*}=c_{m}^{*}$. Thus $b$ is in $\mathcal{C}$.

The lemma follows by noting that $\Gamma=|\mathcal{D}| / 2^{2 m q n}=2^{-m q n} *\left(|\mathcal{C}| / 2^{m q n}\right)$.

Lower bounding the right hand side of the above lemma is a much easier task, as there is no function $f$ involved.

We will denote by $\Delta$ the quantity $(m q)^{2} 2^{-(n+1)}$. 
Lemma 4. For every qn bit constant $r$,

$$
\operatorname{Pr}_{c \in \mathcal{U}\{0,1\} \text { mqn }}\left[P D(\rho(c)) \mid c_{m}^{*}=r\right] \geq 1-\Delta
$$

Proof: First note that for all $i, c_{m}^{i}=\rho(c)_{m}^{i}$, by lemma 2(a) and definition of $\rho$. Thus, once $c_{m}^{*}$ is fixed (and hence $\rho(c)_{m}^{*}$ ) to $r$, the plaintext $p=P(c)$ is fixed, independent of other $c_{j}^{i}(i \in[1 . . q], j<m)$. We will prove the lemma by upper bounding the probability of $\neg \mathrm{PD}$ by union bound.

For each vertex $j$, let $V_{j}$ be its set of incident vertices, i.e. $V_{j}=\{u \mid E(u, j)\}$. Recall,

$$
M_{j}^{i}(\rho(c))=p_{j}^{i}+\sum_{u: E(u, j)} \chi(\langle u, j\rangle) * c_{u}^{\mu_{c}(i, u)}
$$

If $j \neq j^{\prime}$, and $V_{j} \neq V_{j^{\prime}}$, wlog let $w \in V_{j}$ and $w \notin V_{j^{\prime}}$. Then $M_{j}^{i}(\rho(c))=M_{j^{\prime}}^{i^{\prime}}(\rho(c))$ iff

$$
\begin{aligned}
& \chi(\langle w, j\rangle) * c_{w}^{\mu_{c}(i, w)} \\
& =p_{j}^{i}+p_{j^{\prime}}^{i^{\prime}}+\sum_{u: E(u, j), u \neq w} \chi(\langle u, j\rangle) * c_{u}^{\mu_{c}(i, u)}+\sum_{u: E\left(u, j^{\prime}\right)} \chi\left(\left\langle u, j^{\prime}\right\rangle\right) * c_{u}^{\mu_{c}\left(i^{\prime}, u\right)}
\end{aligned}
$$

Since, $c_{w}^{\mu_{c}(i, w)}$ does not appear on the RHS, and $w<m$, and $\chi(\langle w, j\rangle) \neq 0$, the probability of above is $2^{-n}$.

If $j \neq j^{\prime}$, and $V_{j}=V_{j^{\prime}}$, then for some $w \in V_{j}, \chi(\langle w, j\rangle) \neq \chi\left(\left\langle w, j^{\prime}\right\rangle\right)$, as the underlying graph $G$ is non-singular. Thus, similarly to the argument above, $M_{j}^{i}=M_{j^{\prime}}^{i^{\prime}}$ happens with probability $2^{-n}$.

When $j$ equals $j^{\prime}$ (and $i \neq i^{\prime}$ ), we have three cases. If for some $u$ incident on $j(E(u, j)), \mu_{c}(i, u) \neq \mu_{c}\left(i^{\prime}, u\right)$, then the probability of the two $M$ s being equal is at most $2^{-n}$. Otherwise, if $p_{j}^{i} \neq p_{j}^{i^{\prime}}$, then the probability is zero. If $p_{j}^{i}=p_{j}^{i^{\prime}}$, we have $\mu_{c}(i, j)=\mu_{c}\left(i^{\prime}, j\right)$ by lemma $2(\mathrm{j})$, and hence the corresponding disjunct in $\neg \mathrm{PD}$ is false.

Since all the probabilities are $2^{-n}$ or zero, the bound in the lemma follows.

\section{Lemma 5}

$$
\operatorname{Pr}_{f}[\mathrm{PD}(C(f))] \geq 1-\Delta
$$

Proof:

$$
\begin{aligned}
& \operatorname{Pr}_{f}[\operatorname{PD}(C(f))] \\
& =\sum_{r} \sum_{c} \operatorname{Pr}_{f}\left[C(f)=c \wedge \operatorname{PD}(c) \wedge c_{m}^{*}=r\right] \\
& =\sum_{r} \operatorname{Pr}_{c, f}\left[C(f)=c \wedge \operatorname{PD}(c) \wedge c_{m}^{*}=r\right] * 2^{m q n} \\
& =\sum_{r} \operatorname{Pr}_{c, f}\left[C(f)=c \wedge \operatorname{PD}(c) \mid c_{m}^{*}=r\right] * 2^{-q n} * 2^{m q n} \\
& =\sum_{r} 2^{-q n} * \operatorname{Pr}_{c}\left[\operatorname{PD}(\rho(c)) \mid c_{m}^{*}=r\right] \quad \text { (by lemma 1) } \\
& \geq 1-\Delta \quad(\text { by lemma 4) }
\end{aligned}
$$


Since the adversary A decides 0 or 1 based on the oracle replies, say $O=$ $\left\langle O^{1}, O^{2}, \ldots, O^{q}\right\rangle$, we can write its output as $\mathrm{A}(O)$. In scenario (a), $O$ is really $C_{m}^{*}(f)$, with $f$ chosen randomly. Since in scenario (b), the oracle is a random function with range $n$ bits, $O$ is just a uniformly random string of length $q n$.

\section{Lemma 6}

$$
\operatorname{Pr}_{(b)}[A(O)=0] \geq \operatorname{Pr}_{f}\left[A\left(C_{m}^{*}\right)=0 \wedge \operatorname{PD}(C(f))\right] \geq(1-\Delta) \operatorname{Pr}_{(b)}[A(O)=0]
$$

Proof: To begin with, we have

$$
\begin{aligned}
& \operatorname{Pr}_{f}\left[A\left(C_{m}^{*}\right)=0 \wedge \operatorname{PD}(C(f))\right]=\sum_{c} \operatorname{Pr}_{f}\left[A\left(c_{m}^{*}\right)=0 \wedge C(f)=c \wedge \operatorname{PD}(c)\right] \\
& =2^{m q n} * \operatorname{Pr}_{c \in \mathcal{U}\{0,1\}^{m q n}, f}\left[A\left(c_{m}^{*}\right)=0 \wedge C(f)=c \wedge \operatorname{PD}(c)\right] \\
& =2^{m q n} * \operatorname{Pr}_{c \in \mathcal{U}\{0,1\}^{m q n}, f}\left[C(f)=c \wedge \operatorname{PD}(c) \mid A\left(c_{m}^{*}\right)=0\right] * \operatorname{Pr}_{(b)}[A(O)=0]
\end{aligned}
$$

The above is at least $(1-\Delta) \operatorname{Pr}_{(b)}[A(O)=0]$ by lemma 1 and lemma 4 , and at most $\operatorname{Pr}_{(b)}[A(O)=0]$.

Proof of Theorem 2 (Main Theorem): By lemma 6 and lemma 5 it follows that

$$
\left|\operatorname{Pr}_{f}\left[A\left(C_{m}^{*}(f)\right)=0\right]-\operatorname{Pr}_{(b)}[A(O)=0]\right| \leq \Delta
$$

\section{Variable Length Domain Extension and Family of Graphs}

The previous constructions were devoted to extending the domain of a function from $n$ bits to $m n$ bits, for a fixed $m$. In other words, the plaintext queries of the adversary were restricted to be exactly $m n$ bits. We could fix $m$ to be large enough, say $m=2^{n}$, and use a canonical encoding of smaller sized plaintexts into length $m n$ bit strings. Such an encoding exists for all plaintexts of size less than $m n$ by appending plaintexts of size $q$ bits, by $10^{i}$, where $i=m n-q-1$. In other words, $10^{i}$ acts as an end marker. However, smaller sized plaintexts have to undergo $m=2^{n}$ applications of $F$, which is very inefficient. This problem of a really long end marker was resolved by [23] (also see [7]) by noting that the end marker can actually be of length zero, if it can be authenticated.

The simplest way to achieve this is to have two independent PRFs F1 and $F 2$. Use $F 1$ when the plaintext is not a multiple of the block size $n$, and use $F 2$ when the plaintext is a multiple of $n$. In the former case, append an end marker of the kind $10^{i}$, but now $i$ need only be of length at most $n-2$.

So, given a function $F_{k}$ on $n$ bits, consider a collection of graphs, one graph $G_{q}$ in the family for each (plaintext) bit-length $q$. Then if we define $\tilde{F}_{k}^{G_{q}}$ similarly to as before, we have a composite function from all strings to $n$ bits. We know that individually each $\tilde{F}^{G_{q}}$ is a PRF given $F$ is a PRF. As explained in the previous paragraph, we need to assure that these different functions are (almost) 
independent. We prove that if the family of graphs satisfy certain constraints then this is indeed the case.

We consider a fixed $n$ throughout the rest of this section. We will assume that we are only interested in domain extension up to length $2^{n} * n$ bits, as theorem 2 is ineffective beyond that length (this restriction is only for sake of simplicity). Each query of the adversary will be a string $p$ of length $q$ bits, $\left(0<q<2^{n} * n\right)$. We let the composite function answers the query as follows: If $q$ is a multiple of $n$, then it returns $f^{G_{q}}(p)$. Otherwise, let $p^{\prime}$ be $p$ appended with $10^{i}$, where $i$ is the smallest positive number to make $\left|p^{\prime}\right|$ a multiple of $n$. The composite function then returns $f^{G_{\left|p^{\prime}\right|}}\left(p^{\prime}\right)$.

For every $0 \leq l<2^{n}$, since strings of length $l n+1$ to $l n+n-1$ bits get canonically encoded in the above method, we can use the same graph for all these lengths. Thus, for each $l$, we really need only two graphs $([7])$, one for lengths $l n+1$ to $l n+n-1$, and one for length $l n+n$. From now on, we will assume that all plaintexts are of bit length multiples of $n$. Each adversarial query will be a pair: $(p, z)$, where $p$ is a bit string of length multiple of $n$, and $z$ is in $\{0,1\}$ ( $z$ signifies if the plaintext was of length a multiple of $n$ or if it was padded to make it so).

Definition 10. Let $S$ be the set of all binary strings of length non-zero multiples of $n$, but less than $2^{n} * n$. Let $\mathcal{F}$ be the set of all functions:

$$
S \times\{0,1\} \rightarrow\{0,1\}^{n}
$$

Let $\tilde{F}$ be a function with signature:

$$
\{0,1\}^{k} \times S \times\{0,1\} \rightarrow\{0,1\}^{n}
$$

Given a PRF $F$ from $n$ bits to $n$ bits, we need to define $\tilde{F}$ such that no adaptive adversary can distinguish between $\tilde{F}_{K}$, with $K$ chosen randomly, and a function chosen uniformly at random from $\mathcal{F}$. As in the previous sections, given a function $f$ from $n$ bits to $n$ bits, and given a collection of graphs $\mathcal{G}$, we first define a function $f^{\mathcal{G}}$ in $\mathcal{F}$.

Definition 11. Let $\mathcal{G}$ be a collection of edge-colored DAGs $G(l)$ (see definition $5), l \leq\left(2^{n}-1\right) * 2$. Each $G(l)$ is required to have unique source and sink nodes. Further, each $G(l)$ is required to have at least $\left\lceil\frac{l}{2}\right\rceil$ nodes. Define a function $f \mathcal{G}$ as follows:

$$
f^{\mathcal{G}}(p, z)=f^{G(2 *|p|-z)}(p)
$$

where $f^{G}$ is as in definition 6 . If the graph has more nodes than the length of the plaintext, then append enough zeroes to the plaintext. Usually, graphs will have exactly the required number of nodes. However, at the base cases, i.e. small length plaintexts, it may be necessary to have extra nodes. For an example, see Section 5.1.

For a theorem similar to theorem 2 to hold, we need further restrictions on $\mathcal{G}$. In particular, it will not be enough that individual graphs in $\mathcal{G}$ be nonsingular. Since, we will need to extend the notion of non-singularity to the whole 


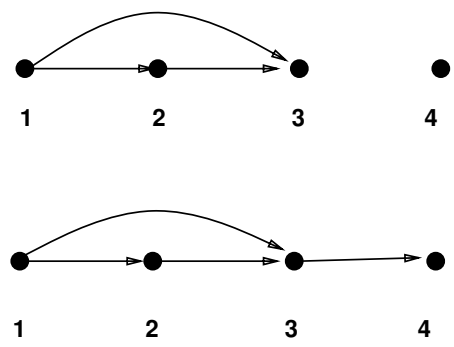

Fig. 5. An Incorrect Construction

collection of graphs, it is best to fix a set of vertices $V$, and just define the edges and colorings for the individual graphs. Thus, we will define $E(l)$, and $\chi(l)$. The partial order $\prec_{l}$ is, as before, the transitive closure of $E(l)$.

To motivate the generalized definition of non-singularity, we first consider an example where it is not enough for individual graphs to be non-singular. Let $V=[1 . .4]$. The graphs are identical (see fig 5), except that the second graph $G(2)$ has an extra edge from 3 to 4 . The first graph $G(1)$ is used to answer queries of length 3 blocks, and the second to answer queries of length 4 . Clearly, both graphs are individually non-singular. Consider two queries, one of length three, and another of length four, the latter being just an extension of the first. However, the first graph's output is $C_{3}$, and is accessible to the adversary. Thus, during the second query the internal state $C_{3}$ is available to the adversary, and it can force $M_{4}$ to be any value of its choice.

This suggests that for each graph $G(i)$, the graph $G(i)$ itself cannot be allowed to be an induced subgraph of another graph $G\left(i^{\prime}\right)$. We prove that this condition is sufficient for the composite function to be a PRF.

Definition 12. For any vertex $j$ in $V$, let $U_{j}^{l}$ be the set of incident vertices of $j$ in $G(l)$.

For any vertex $j$ in $V$, we say $(l, j) \cong\left(l^{\prime}, j\right)$ if either $(j=1)$ or

- $U_{j}^{l}=U_{j}^{l^{\prime}}$, and

- for all $u \in U_{j}^{l}: \chi_{l}(\langle u, j\rangle)=\chi_{l^{\prime}}(\langle u, j\rangle)$, and inductively $(l, u) \cong\left(l^{\prime}, u\right)$. Essentially, $(l, j)$ is congruent to $\left(l^{\prime}, j\right)$ if the two graphs $G(l)$ and $G\left(l^{\prime}\right)$ are identical till $j$.

Definition 13. Let $\mathcal{G}=\langle G(l)\rangle$, where each $G(l)=(V, E(l), \chi(l))$ is an edgecolored DAG, be a collection of graphs.

- With each $G(l)$ we associate its size $m(l)$ to be the largest numbered node in $V$ such that there is an edge directed to it in $G(l)$.

- For each $G(l)$ we define the graph $\tilde{G}(l)=([1 . . m(l)], E(l), \chi(l))$, to be the induced subgraph of $G(l)$ on vertices $[1 . . m(l)]$.

The collection $\mathcal{G}$ is called PRF-preserving if

- each $\tilde{G}(l)$ has only one source node, one sink node, has at least $\left\lceil\frac{l}{2}\right\rceil$ nodes, and 
- if for any pair of nodes $u, v(u \neq v)$, and graphs $G(l)$ and $G\left(l^{\prime}\right)$, the set of incident nodes of $u$ in $G(l)$, and the set of incident nodes of $v$ in $G\left(l^{\prime}\right)$ are same (say $W)$, then for at least one $w \in W, \chi_{l}(\langle w, u\rangle) \neq \chi_{l^{\prime}}(\langle w, v\rangle)$.

- for each graph $G(l)$, it is not the case that there is another graph $G\left(l^{\prime}\right)$, $l^{\prime} \neq l$, s.t. $\left(l, m\left(l^{\prime}\right)\right) \cong\left(l^{\prime}, m\left(l^{\prime}\right)\right)$

Basically, the second condition above has extended the non-singularity requirement to be over all graphs.

Theorem 4. For a PRF-preserving collection of $2 *\left(2^{n}-1\right)$ DAGs $\mathcal{G}$, let $f^{\mathcal{G}}$ be as in definition 11. Then, no adaptive adversary, with $q$ adaptive queries $\left\langle\left(p^{i}, z^{i}\right)\right\rangle$ $\left(i \in[1 . . q]\right.$, and $\left.\left|p^{i}\right| \leq 2^{n}-1\right)$, can distinguish between (a) $f^{\mathcal{G}}$ where $f$ is chosen uniformly at random from $\mathcal{F}(n \rightarrow n)$, (b) and a function chosen uniformly at random from $\mathcal{F}$, with probability more than $\left(\sum_{i \in[1 . . q]}\left|p^{i}\right|\right)^{2} 2^{-(n+1)}$.

Proof: To adapt the proof of theorem 2, we first need to redefine the notion of consistent transcripts $c$. First note that, on a fixed transcript $c$, the queries of the adversary are fixed, say $\left\langle p^{i}, z^{i}\right\rangle_{i \in[1 . . q]}$. Recall, by definition of $f \mathcal{G}$, on input $p^{i}, z^{i}$ the graph $G\left(2 *\left|p^{i}\right|-z^{i}\right)$ is used. We just denote this graph by $G^{i}$. The corresponding edge relation, coloring and partial order will be denoted $E^{i}, \chi^{i}$, and $\prec^{i}$ resp. Also, for the graph $G^{i}$, its induced subgraph as per definition 13, will be denoted $\tilde{G}^{i}$. Similarly, the size of the graph $\tilde{G}^{i}$ will be denoted by $m^{i}$. Note that $m^{i}=\left|c^{i}\right| \geq\left|p^{i}\right|$.

Definition 14. For any vertex $j$ in $V$, let $V_{j}^{i}$ be the set of incident vertices of $j$ in $G^{i}$.

For any vertex $j$ in $V$, we say $(i, j) \cong{ }_{c}\left(i^{\prime}, j\right)$ if either $(j=1)$ or - $V_{j}^{i}=V_{j}^{i^{\prime}}$, and

- for all $u \in V_{j}^{i}: \chi^{i}(\langle u, j\rangle)=\chi^{i^{\prime}}(\langle u, j\rangle)$, and inductively $(i, u) \cong_{c}\left(i^{\prime}, u\right)$.

Essentially, $(i, j)$ is congruent (w.r.t. $c)$ to $\left(i^{\prime}, j\right)$ if the two graphs $G^{i}$ and $G^{i^{\prime}}$ are identical till $j$.

Once we generalize the definition of $\equiv_{c}$, rest of the definitions and proofs remain almost the same.

Definition 15. For any vertices $j, j^{\prime}$, and query indices $i, i^{\prime}$ we say that $(i, j) \equiv_{c}$ $\left(i^{\prime}, j^{\prime}\right)$ if

$$
\left(j=j^{\prime}\right) \text { and }(i, j) \cong_{c}\left(i^{\prime}, j\right) \text { and } \forall k \preceq^{i} j: p_{k}^{i}=p_{k}^{i^{\prime}}
$$

As before, define

$$
\mu_{c}(i, j)=\min \left\{i^{\prime} \mid\left(i^{\prime}, j\right) \equiv_{c}(i, j)\right\} .
$$

We call $c$ consistent $(\operatorname{con}(c))$ if

$$
\forall j \in\left[1 . .2^{n}-1\right], \forall i: c_{j}^{i}=c_{j}^{\mu_{c}(i, j)}
$$

Define the following "correcting" function $\rho$ :

$$
\rho(c)=\bar{c}, \text { where } \bar{c}_{j}^{i}=c_{j}^{\mu_{c}(i, j)}, \text { for } j \in\left[1 . . m^{i}\right]
$$


Since the proof of theorem 10 will be adapted from the proof of theorem 2, we will denote all lemmas for theorem 10 corresponding to lemmas for theorem 2 by the prime symbol. In the proof of lemma $2(\mathrm{a})^{\prime}$, if $m^{i} \neq m^{i^{\prime}}$, then $(i, m) \approx_{c}\left(i^{\prime}, m^{\prime}\right)$. Otherwise, if the plaintexts $p^{i}$ and $p^{i^{\prime}}$ are different, then again $\left(i, m^{i}\right) \not \equiv_{c}\left(i^{\prime}, m^{i}\right)$. If the plaintexts are also same, then as the adversary does not repeat queries, wlog let $G^{i}=G\left(2 * m^{i}-1\right)$, and $G^{i^{\prime}}=G\left(2 * m^{i}\right)$. But $\left(i, m^{i}\right) \cong_{c}\left(i^{\prime}, m^{i}\right)$ is not allowed in $\mathcal{G}$ which is PRF-preserving. That proves lemma $2(\mathrm{a})^{\prime}$.

Proof of rest of lemma $2^{\prime}$ is similar to proof of lemma 2 . In the statement and proof of lemma $2(\mathrm{f})^{\prime}, j$ must be restricted to be $\left[1 . . m^{i}\right]$. Similar restrictions apply in the definition of PD (definition 8) and definition of $f_{c}$ (definition 9 ). Proof of lemma $3^{\prime}$ is similar to proof of lemma 3 .

Lemma 1 is now restated as (recall $\mathrm{S}$ from definition 10):

Lemma $\mathbf{1}^{\prime}$. For every $q n$ bit constant $\left\langle r^{i}\right\rangle(i \in[1 . . q])$

$$
\begin{aligned}
& \operatorname{Pr}_{c \in_{U} S^{q}, f}\left[C(f)=c \wedge \operatorname{PD}(c) \mid \forall i: c_{m^{i}}^{i}=r^{i}\right] \\
& =2^{-m q n} * \operatorname{Pr}_{c \in_{U} S^{q}}\left[\operatorname{PD}(\rho(c)) \mid \forall i: c_{m^{i}}^{i}=r^{i}\right]
\end{aligned}
$$

Proof Sketch: The proof is similar to proof of lemma 1, if we notice that we fix $c$ in the first part of the proof. For a fixed $c$, let $I=\left\{(i, j) \mid \mu_{c}(i, j)=(i, j), j \in\right.$ $\left.\left[1 . . m^{i}\right]\right\}$. Let $T=\left\{M_{j}^{i}(c) \mid(i, j) \in I\right\}$. Since PD(c) holds, $|T|=|I|$. Let $T^{\prime}$ be an arbitrary set of $n$ bit strings, disjoint from $T$, and $\left|T^{\prime}\right|=\sum_{i \in[1 . . q]} m^{i}-|I|$. Thus, $\left|T \cup T^{\prime}\right|=\sum_{i \in[1 . . q]} m^{i}$.

By, lemma $3^{\prime}, C\left(f_{c}\right)=c$. Thus, for each $b$ agreeing with $c$ on $I$, we have a function $f_{c}$ defined on $|I|$ inputs $T$, such that $C\left(f_{c}\right)=c$. We can use the remaining $\sum_{i \in[1 . . q]} m^{i}-|I|$ values of $b$ (i.e. from indices which are not in $I$ ) to extend $f_{c}$ to be defined on $T \cup T^{\prime}$. This map from $b$ to the extended $f_{c}$ is $1-1$.

The reverse direction is done as in lemma 1.

Rest of the proof is also as in proof of lemma 1.

Let $\Delta$ denote $\left(\sum_{i \in[1 . . q]} m^{i}\right)^{2} * 2^{-(n+1)}$.

Lemma $7^{\prime}$. For every $q n$ bit constant $\left\langle r^{i}\right\rangle(i \in[1 . . q])$,

$$
\operatorname{Pr}_{c \in \mathcal{U} S^{q}}\left[\operatorname{PD}(\rho(c)) \mid c_{m^{i}}^{i}=r^{i}\right] \geq 1-\Delta
$$

Proof: First note that for all $i, c_{m^{i}}^{i}=\rho(c)_{m^{i}}^{i}$, by lemma $2(\mathrm{a})^{\prime}$ and definition of $\rho$. As opposed to lemma 4 , we need to show that it is not the case that a $\rho(c)_{j}^{i}$, with $j \neq m^{i}$, can be defined to be a $c_{j}^{i^{\prime}}$, such that $j=m^{i^{\prime}}$. Suppose, there is indeed an $\left(i^{\prime}, j\right) \equiv_{c}(i, j)$, such that $j=m^{i^{\prime}}$. Since, $\left(i^{\prime}, j\right) \equiv_{c}(i, j)$, we have $\left(i^{\prime}, j\right) \cong_{c}(i, j)$. Thus the graphs $G^{i}$ and $G^{i^{\prime}}$ are identical till $j=m^{i^{\prime}}$. Thus, unless they are the same graph, this is not allowed by the condition on PRF-preserving $\mathcal{G}$. If they are the same graph, then $j=m^{i}$, a contradiction.

Rest of the proof is similar to proof of lemma 4.

Rest of the proof of theorem 10 is identical to that of theorem 2 . 


\subsection{Applications to Variable Length Domain Extension}

As an application of theorem 10, we get the variable length domain extension scheme as described in figure 6 . In the figure, for each plaintext block length two graphs are given as required in definition 11. The number on the left of the graphs denotes the plaintext block lengths for which those graphs are to be employed. The $0 / 1$ bit signifies if the plaintext was padded to make its bit-length a multiple of $n$. We have only illustrated graphs up to length five, as for larger lengths, we follow similar methods as for length four and five. Note that for plaintext block length one, we have graphs which have two nodes. As remarked at the end of definition 11, this requires that plaintexts of length one block must be appended with a zero block, before employing graphs "ONE-0" or "ONE-1".

This mode has an advantage over XCBC [7], and OMAC [14] that it does not even need to employ the initial $F$ on a constant like $0^{n}$. Moreover, the scheme shows that if the plaintexts are restricted to be more than three blocks in length, then no Galois field arithmetic is required.

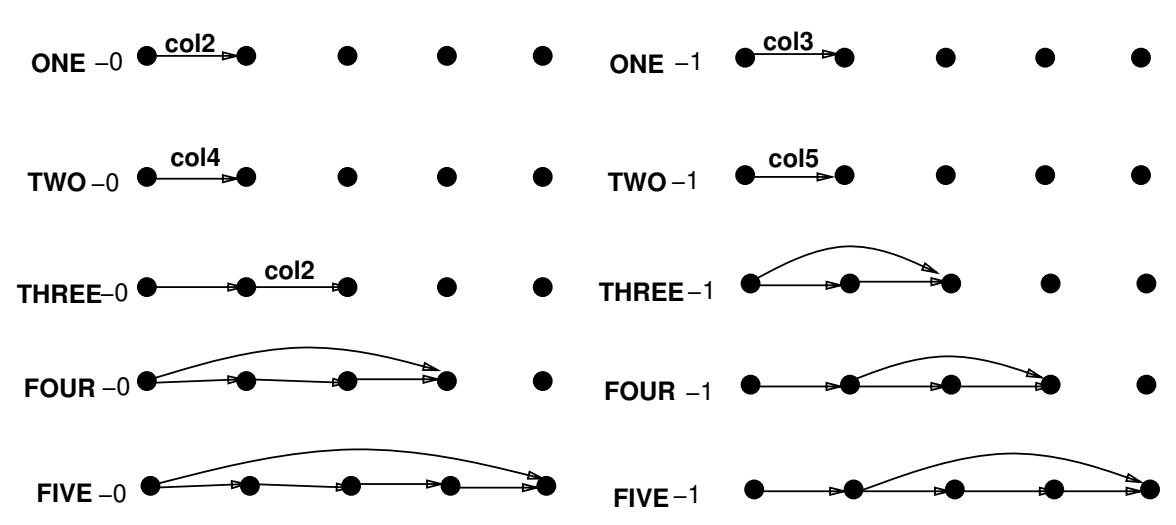

Fig. 6. A Variable Length Mode

\section{References}

1. ANSI X3.106, "American National Standard for Information Systems - Data Encryption Algorithm - Modes of Operation", American National Standards Institute, 1983.

2. M . Bellare, R. Canetti, H. Krawczyk, "Pseudorandom Functions Revisited: The Cascade Construction and its Concrete Security", Proc. IEEE FOCS 1996.

3. M. Bellare, J. Kilian, P. Rogaway, "The Security of Cipher Block Chaining", JCSS, Vol. 61, No. 3, Dec 2000, pp. 362-399

4. M. Bellare, A. Desai, E. Jokiph, P. Rogaway, "A Concrete Security Treatment of Symmetric Encryption: Analysis of the DES Modes of OPeration", 38th IEEE FOCS, 1997

5. D. Bernstein, "How to Stretch Random Functions: The security of Protected Counter Sums", J. of Cryptology, Vol 12,No. 3, (1999). 
6. J. Black, P. Rogaway, "A Block Cipher Mode of Operation for Parallelizable Message Authentication", Proc. Eurocrypt 2002.

7. J. Black, P. Rogaway, "CBC MACs for arbitrary length messages: The three key constructions". CRYPTO 2000, LNCS 1880.

8. J. Carter, M. Wegman, "Universal Classes of Hash Functions", JCSS, Vol. 18, 1979, pp 143-154.

9. O. Goldreich, S. Goldwasser, and S. Micali, "How to construct random functions", J. ACM, vol. 33, no. 4, 1986.

10. National Bureau of Standards, Data Encryption Standard, U.S. Department of Commerce, FIPS 46 (1977)

11. V.D. Gligor, P. Donescu, "Fast Encryption Authentication: XCBC Encryption and XECB Authentication Modes", http://csrc.nist.gov/encryption/modes/workshop1

12. S. Halevi and P. Rogaway, "A Tweakable Enciphering Mode", CRYPTO 2003, LNCS 2729.

13. F. Harary, Graph Theory, Addison-Wesley 1969.

14. T. Iwata, K. Kurosawa, "OMAC: One -key CBC-MAC", FSE 2003, LNCS 2887.

15. C. S. Jutla, "Generalized Birthday Attacks on Unbalanced Feistel Networks", CRYPTO 1998, LNCS 1462.

16. C. S. Jutla, "Encryption Modes with Almost Free Message Integrity", Proc. Eurocrypt 2001, LNCS 2045, 2001.

17. Hugo Krawczyk, "LFSR-based Hashing and Authentication", Proc. Crypto 94, LNCS 839, 1994

18. H.W. Kuhn, "Extensive games and the problem of information" in Contributions to the Theory of Games II, H.W. Kuhn and A. W. Tucker eds., Annals of Mathematical Studies No. 28, Princeton Univ. Press, 1950.

19. M. Liskov, R. Rivest and D. Wagner, "Tweakable Block Ciphers", CRYPTO 2002, LNCS 2442.

20. M. Luby, "Pseudorandomness and Cryptographic Applications", Princeton Computer Science Notes, Princeton Univ. Press, 1996

21. M. Luby and C. Rackoff, "How to Construct Pseudorandom Permutations From Pseudorandom Functions", SIAM J. on Computing, Vol. 17, 1988, pp. 373-386.

22. M. Naor and O. Reingold, "On the construction of pseudo-random permutations: Luby-Rackoff revisited", Proc. 29th ACM STOC, 1997, pp 189-199.

23. E. Petrank, C. Rackoff, "CBC-MAC for real-time data sources", J. of Cryptology, vol 13, no. 3, nov 2000.

24. P. Rogaway, M. Bellare, J. Black and T. Krovetz, "OCB: A block-cipher mode of operation for efficient authenticated encryption", Proc. 8th ACM Conf. Comp. and Comm. Security (CCS), ACM, 2001.

\section{Appendix}

Lemma 3. For any consistent $c$ such that $\mathrm{PD}(c)$ holds:

$$
C\left(f_{c}\right)=c
$$

Proof: Let $p=P(c)$ and $M=M(c)$ be shorthands. Also, we will use $\bar{c}$ as shorthand for $C\left(f_{c}\right)$. Similarly, let $\bar{M}=M(\bar{c}), \bar{p}=P(\bar{c})$.

We do induction over the query index. 
Base Case: Since the adversary is fixed, the first plaintext message is the same, i.e. $\bar{p}^{1}=p^{1}$. Since $\bar{M}_{1}^{1}=p_{1}^{1}, \bar{c}_{1}^{1}=f_{c}\left(\bar{M}_{1}^{1}\right)=f_{c}\left(M_{1}^{1}\right)=c_{1}^{1}$, as $(1,1)$ is trivially in $I$. For $j>1, \bar{M}_{j}^{1}=p_{j}^{1}+\sum_{u: E(u, j)} \chi(\langle u, j\rangle) * \bar{c}_{u}^{1}$ But, by induction over the partial order $\prec, \bar{c}_{u}^{1}=c_{u}^{1}$, hence $\bar{M}_{j}^{1}=M_{j}^{1}$. Moreover, $(1, j)$ is trivially in $I$, and hence $\bar{c}_{j}^{1}=c_{j}^{1}$.

So, assume that for all $i^{\prime}<i$, and all $j, \bar{c}_{j}^{i^{\prime}}=c_{j}^{i^{\prime}}$. Thus, $\bar{p}^{i}=p^{i}$. Again, $\bar{M}_{1}^{i}=p_{1}^{i}=M_{1}^{i}$. Thus, $\bar{c}_{1}^{i}=f_{c}\left(M_{1}^{i}\right)=f_{c}\left(M_{1}^{\mu_{c}(i, 1)}\right)$ by lemma $2(\mathrm{~h})$. By definition of $f_{c}$, this is same as $c_{1}^{\mu_{c}(i, 1)}=c_{1}^{i}$. For $j>1, \bar{M}_{j}^{i}=p_{j}^{i}+\sum_{u: E(u, j)} \chi(\langle u, j\rangle) * \bar{c}_{u}^{i}$. But, by induction over the partial order $\prec, \bar{c}_{u}^{i}=c_{u}^{i}$, thus $\bar{M}_{j}^{i}=M_{j}^{i}$. As before, using lemma $2(\mathrm{~h})$, we are done. 